\title{
Comparison of BCG vaccination at birth and at third month of life
}

\author{
Íbrahim Ildirim, Nihat Sapan, Bülent Çavuşoğlu
}

\begin{abstract}
Tuberculosis is an important health problem in developing countries and the BCG vaccine plays an important part in preventing the disease. There are different reports about the preventive value of BCG. Some of them claim that it is satisfactory while others suggest that it provides little protection. There are also varying ideas about the optimum time to vaccinate babies, some studies suggesting that late vaccination confers a high degree of protection. This prospective controlled study has been undertaken to evaluate the value of BCG vaccine given to babies during their first three days of life versus its value when given in their third month of life. Evaluation was measured by the results of tests with purified protein derivative (PPD), by vaccine scars, and by the complications of the vaccine. It was found that BCG given at the end of the third month provides a higher rate of response and fewer complications than when given during the first three days of life.
\end{abstract}

BCG plays a significant part in protection against tuberculosis, which is one of the most important problems in developing countries. Nevertheless, the protective effect of vaccine remains a highly controversial subject, and has been reported to range from $80 \%$ to less than $5 \% .^{1-6}$ In many countries the first BCG vaccine is received at birth, but it has been suggested that this requires further evaluation. ${ }^{178} \mathrm{~A}$ controlled prospective study has been recommended $^{17}$ to compare BCG results from vaccination at birth and those at the third month of life. This prospective controlled study was planned to compare tuberculin response, BCG scar, and the complications of the vaccine given in the first three days compared with the third month of life.

Subjects and methods

The subjects selected for the study were healthy, full term babies of over $2500 \mathrm{~g}$ born either in the Uludağ University Medical School Hospital or City Maternity Hospital in Bursa between October 1989 and January 1990. All the babies were followed up to 12 months. Babies who had a history of tuberculosis among their family or close contacts were excluded from the study.

Babies were randomly divided into two groups. Four hundred babies in the first group. (group I) were vaccinated with BCG intradermally in the left shoulder in the first three days of life, and the second group of 400 babies (group II) was vaccinated in the same manner at the end of the third month ( $90 \pm 3$ days) of life. Vaccines were given to both groups by the same trained personnel. At the beginning of the study the mothers of the babies under study were carefully examined and their BCG scars and induration of purified protein derivative (PPD) were recorded. For the babies in group II, a PPD test was carried out before the BCG vaccination. The babies in both groups were examined every three months until they were 12 months old, PPD tests were performed three months after BCG vaccination and at the end of the first year. A full examination was performed in the follow up and BCG complications were recorded. Lymphadenopathies of more than $10 \mathrm{~mm}$ maximum diameter and showing abscess formation and fistulisation were accepted as complications of BCG vaccine. Seventeen babies from the first group and 21 babies from the second group were excluded from the study as they had other diseases.

The same lot number of BCG, from Canada Connaught Laboratory, was used in both groups, and given in $0.05 \mathrm{ml}(0.05 \mathrm{mg})$ doses. PPD from Denmark Statens Seruminstitut (RT-23 Mantoux test) was given intradermally to the volar surface of the forearm in 5 tuberculin units $/ 0 \cdot 1 \mathrm{ml}$ doses. Seventy two hours later the induration caused by PPD was measured horizontally and vertically and the average of the measurements was recorded. During the controlling examinations BCG scar and complications were also measured and recorded.

Statistical comparisons were by Student's $t$ test and $z$ (normal distribution) test between groups.

\section{Results}

The number of babies who came to all the follow up examinations in group I was 313 (78\%) and in group II was $334(84 \%)$. Mean (SD) birth weights were $3370(41) \mathrm{g}$ in group I and 3410 (36) $\mathrm{g}$ in group II; statistical analysis revealed no significant difference. Weight gain was similar in the two groups. The feeding conditions were also similar. There were 169 babies (54\%) in group I and $172(52 \%)$ in group II who had breast feeding only; while 120 babies $(38 \%)$ in group I and $128(38 \%)$ in group II were fed by both formula feed and breast milk. The remaining 24 babies $(8 \%)$ in group $I$ and 34 $(10 \%)$ in group II were fed by formula only. There were no significant differences between the groups.

As is seen in table 1 , when PPD results were compared the diameter of the area of induration 
Table 1 Diameter of area of induration ( $\mathrm{mm}$ ) after the $P P D$ test, comparing BCG given in the first three days (group I) and at the end of the third month (group II). Results are mean (SD)

\begin{tabular}{llll}
\hline & $\begin{array}{l}\text { Group } I \\
(n=313)\end{array}$ & $\begin{array}{l}\text { Group II } \\
(n=334)\end{array}$ & p Value \\
\hline Three months after BCG & $\begin{array}{l}6 \cdot 9(3 \cdot 8) \\
8 \cdot 4(4 \cdot 8)\end{array}$ & $\begin{array}{l}10 \cdot 4(4 \cdot 3) \\
10 \cdot 8(4 \cdot 5)\end{array}$ & $<0 \cdot 001$ \\
\hline Months old & 80.001
\end{tabular}

Table 2 The distribution of cases having PPD results of less than $5 \mathrm{~mm}$ induration. Results are number (\%)

\begin{tabular}{llll}
\hline & Group I & Group II & p Value \\
\hline Three months after BCG & $122(39)$ & $43(13)$ & $<0.001$ \\
12 Months old & $104(33)$ & $43(13)$ & $<0.001$ \\
\hline
\end{tabular}

Table 3 Details of the BCG scar at the end of the 12 months

\begin{tabular}{llll}
\hline & $\begin{array}{l}\text { Group I } \\
(n=313)\end{array}$ & $\begin{array}{l}\text { Group II } \\
(n=334)\end{array}$ & $p$ Value \\
\hline $\begin{array}{l}\text { No (\%) of cases not having scar } \\
\text { Mean (SD) average scar diameter (mm) }\end{array}$ & $\begin{array}{l}22(7) \\
3.6(1.4)\end{array}$ & $\begin{array}{l}2(0.6) \\
4.4(1 \cdot 1)\end{array}$ & $<0.001$ \\
\hline
\end{tabular}

Table 4 Comparison of the BCG complications. Results are number (\%)

\begin{tabular}{lccl}
\hline & Group I & Group II & p Value \\
\hline Lymphadenopathies (more than 10 mm) & $52(16 \cdot 6)$ & $17(5 \cdot 1)$ & $<0 \cdot 001$ \\
Lymphadenopathies with fistulisation & $9(2 \cdot 9)$ & $8(2 \cdot 4)$ & NS \\
\hline
\end{tabular}

is significantly larger after vaccination in the third month compared with those vaccinated in the newborn period; this was true for the comparison made three months after vaccination and also at the age of 12 months.

The distribution of those PPD results that were less than $5 \mathrm{~mm}$ in diameter are shown in table 2. The diameter of area of induration was significantly smaller in the babies that were vaccinated in the first three days of life compared with those vaccinated at three months.

The comparison of the BCG scars (table 3 ) shows that there were fewer and smaller scars in group I compared with group II.

As is seen in table 4, the number of lymphadenopathies with a diameter of more than 10 $\mathrm{mm}$ was found to be less in group II than in group I.

\section{Discussion}

For the prevention of tuberculosis, early detection and treatment of the cases and finding their contacts are essential. To eliminate the disease it is necessary to improve living conditions and use chemoprophylaxis or BCG vaccination. Eighty per cent of the world's population is living in the developing countries and it is very hard to improve their living conditions. For this reason BCG vaccination is accepted as one of the most important protective measures against tuberculosis. It is compulsory in 64 countries and is officially recommended in the remaining 118 countries and territories. ${ }^{17}$ Although BCG has been used for more than 60 years, its protective value is still under discussion. Studies have shown that the efficacy of the vaccine varies between 5 and $80 \% .^{16}$ Rosenthal et al state 'The usefulness of vaccination has been questioned on the basis that since the majority of cases of tuberculosis occur among the tuberculin positive individuals, and that, therefore BCG vaccination of the tuberculin negative portion of the population would not exert much influence on the incidence of disease. This misses the point that, had the tuberculin positive individuals been vaccinated prior to the time of their initial exposure to virulent tubercle bacilli, the incidence of tuberculous disease in this group might have been reduced to an appreciable degree. This situation actually provides a strong argument for the vaccination of infants and children, in areas of high incidence, against tuberculosis, with revaccination at suitable intervals in order to maintain as high a level of immunity as possible." It can be postulated that in developing countries BCG still plays an important part in the control of tuberculosis. Today BCG is used in the neonatal period in many countries. But there is little information on the efficacy of BCG vaccination in the newborn. In a previous study conducted in England, 149 Asian children who received BCG vaccine shortly after birth were reviewed at the age of 22 months and half of them had a negative response to tuberculin. ${ }^{7}$

The immunity provided by BCG is controlled with the PPD-S test 6-8 weeks after vaccination. Since implementation and validation of the PPD test is a simple procedure, it provides an easy assessment of acquired and/or natural immunity of individuals or populations. Our study has shown that when BCG is given at the end of the third month a better PPD response is obtained than when it is given in the first three days of life. The proportion of cases that did not produce a BCG scar is much less in those vaccinated at the end of the third month.

Certain complications of vaccination such as suppurative adenitis and BCG osteomyelitis are more common in newborn infants. ${ }^{10}$ In some recent immunisation programmes BCG has been given a few months after birth. Studies are being carried out to establish whether vaccination induces a better immune response with less risk of complications at this age rather than during the newborn period. ${ }^{7}$

In this study the proportion of the lymphadenopathies larger than $10 \mathrm{~mm}$ diameter was found to be more in the babies who were vaccinated in the first three days than in those who were vaccinated at the end of the third mohth of life. The proportion of lymphadenopathies showing abscess formation and fistulisation was found to be similar in both groups.

We gave no treatment for complications except in the case of lymphadenopathies where abscesses were aspirated and fistulae dressed.

Our results show that BCG given at the end of the third month is more effective and gives a higher rate of PPD response and fewer complications of lymphadenopathy than BCG given in the first three days of life. According to these findings, it is recommended that practice be change in those countries in which BCG is given to newborn infants, and that the vaccine be given at the end of the third month instead. As our study shows that BCG vaccination at the third month gives more strongly positive 
Mantoux tests at the end of the first year, our expectation is that protection from tuberculosis will be more powerful. This assumption is based on the fact that these babies will have a stronger cellular immunity. The prevention of tuberculosis should be the next subject for evaluation. We hope that the further studies will help to find the optimum time for BCG vaccination to provide the best immunity.

This study was supported by the Uludağ University Research Fund (1989/6).

1 Price JF. BCG vaccination. Arch Dis Child 1982;57:485-6.

2 Stein SC, Aronson JD Occurrence of pulmonary lesions in BCG-vaccinated and unvaccinated persons. Am Rev Tuberc 1953;68:695-726.
3 Comstock GW, Palmer CE. Long terms results of BCG vaccination in the southern United States. Am Rev Respir Dis 1966;93:171-83.

4 Clemens J, Chuong J, Feinstein A. The BCG controversymethodological and statistical reappraisal. $\mathcal{F A M A} 1983$; 249:2362-9.

5 Houston S, Fanning A, Soskolne CL, Fraser N. The effectiveness of Bacillus Calmette-Guérin (BCG) vaccination against tuberculosis - a case-control study in Treaty Indians, Alberta, Canada. Am $\mathcal{F}$ Epidemiol 1990;131:340-8.

6 Tidjani $\mathrm{O}$, Amedome A, ten Dam $\mathrm{H}$. The protective effect of $B C G$ vaccination of the newborn against childhood tuberculosis in an African community. Tubercle 1986;67:269-81. 7 Grindulis H, Baynham MID, Scott PH, Thompson RA Wharton BA. Tuberculin response two years after BCG vaccination at birth. Arch Dis Child 1984;59:614-9.

8 Anonymous. BCG vaccination in the newborn (editorial). $B M \mathcal{F} 1980 ; 281: 1445-6$.

9 Rosenthal S, Loewinsohn E, Graham ML, et al. BCG vaccination against tuberculosis in Chicago. Pediatrics vaccination against

10 Victoria MS, Shan BS. Bacillus Calmette-Guérein. Lymphadenitis: a case report and review of the literature. Pediatr adenitis: a case report and
Infect $D$ is $1985 ; 4: 295-6$. 